\title{
Hard-landing Simulation by a Hierarchical Aircraft Landing Model and an Extended Inertia Relief Technique
}

\author{
Kyu Beom Lee*, Seon Ho Jeong*, Jin Yeon Cho** and Jeong Ho Kim*** \\ Department of Aerospace Engineering, Inha University, 100 Inha-Ro, Nam-Gu, Incheon 22212, Korea

\section{Chan Yik Park****} \\ Aeronautical Technology Directorate, Agency for Defense Development, Yuseong-Gu, P.O. Box 35-7, Daejeon 34188, Korea
}

\begin{abstract}
In this work, an efficient aircraft landing simulation strategy is proposed to develop an efficient and reliable hard-landing monitoring procedure. Landing stage is the most dangerous moment during operation cycle of aircraft and it may cause structural damage when hard-landing occurs. Therefore, the occurrence of hard-landing should be reported accurately to guarantee the structural integrity of aircraft. In order to accurately determine whether hard-landing occurs or not from given landing conditions, full nonlinear structural dynamic simulation can be performed, but this approach is highly timeconsuming. Thus, a more efficient approach for aircraft landing simulation which uses a hierarchical aircraft landing model and an extended inertia relief technique is proposed. The proposed aircraft landing model is composed of a multi-body dynamics model equipped with landing gear and tire models to extract the impact force and inertia force at touch-down and a linear dynamic structural model with an extended inertia relief method to analyze the structural response subject to the prescribed rigid body motion and the forces extracted from the multi-body dynamics model. The numerical examples show the efficiency and practical advantages of the proposed landing model as an essential component of aircraft hard-landing monitoring procedure.
\end{abstract}

Key words: Structural integrity, Hard-landing monitoring, Landing simulation, Inertia relief

\section{Introduction}

During takeoff and landing of aircraft, various types of accidents may happen. Those include runway over-run, excursions off the sides of runways on landing, landing gear failures, controlled flight into terrain, and hard-landing. Among these various types of accidents, hard-landing is the most common accident which occurs during landing of aircraft and actually it happened 54 times of 395 commercial jets over 60,000 pounds from 1993 to 2002 [1]. Although hardlanding itself does not result in fatality, it may cause damage to airframe which leads to catastrophic accident during the next flight because invisible micro crack developed by hard-landing can grow to macro structural damage in the case of long- term operating aircraft [2]. Therefore determining whether hard-landing occurs or not is certainly very significant task for safety. Examples of damage to airframe caused by hardlanding accident are shown in Fig. 1[3] and 2[4].

However it is not easy to determine whether hard-landing occurs or not because objective guidelines are not set up at the present time. In Boeing, for example, sink rate estimation is used to determine hard-landing. On the other hand, maximum landing weight prescribed in JARs(European Joint Airworthiness Requirements) and FARs(U.S. Federal Aviation Regulations) is used in Airbus structural team.

In spite of these guidelines, the pilots or crews generally judge hard-landing by their intuitive inspection [5]. If a pilot makes a conclusion that hard-landing occurs, he will submit
This is an Open Access article distributed under the terms of the Creative Commons Attribution Non-Commercial License (http://creativecommons.org/licenses/by$\mathrm{nc} / 3.0 /$ which permits unrestricted non-commercial use, distribution, and reproduction in any medium, provided the original work is properly cited.

\footnotetext{
(c) * Graduate Student

** Professor, Corresponding author: cjy@inha.ac.kr

*** Professor, E-mail: JeongHoKim@inha.ac.kr

**** Principal Researcher, E-mail: pcy1216@add.re.kr
} 
a report about it and then structural examination will be performed. Generally 1st step is visual inspection in 1 and half hours and 2nd step is Non Destructive Examination (NDE) inspection in 8 hours or over. As a result of the examination, there are direct cost corresponding to repair expenses and indirect cost incurred by not operating the aircraft while it is stuck in repair facility. Therefore hard-landing should be carefully reported in order to reduce unnecessary cost.

However, according to the Boeing Company's report illustrated in Fig. 3, current portion of wrong prediction is around $85 \%$ [6]. One of the causes of this high percentage is that pilots tend to report hard-landing conservatively

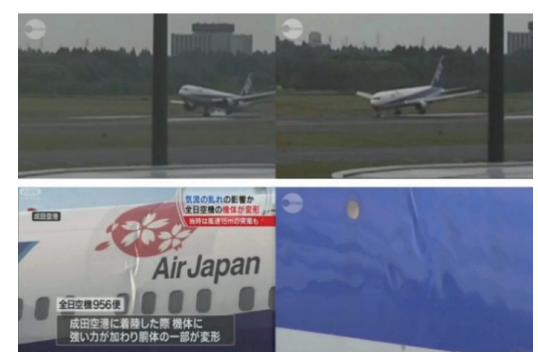

Fig. 1. All Nippon Airways Boeing 767381 ER sustained substantial damage in a hard-landing accident at Tokyo-Narita Airport (NRT), Japan on June 20, 2012. [3]

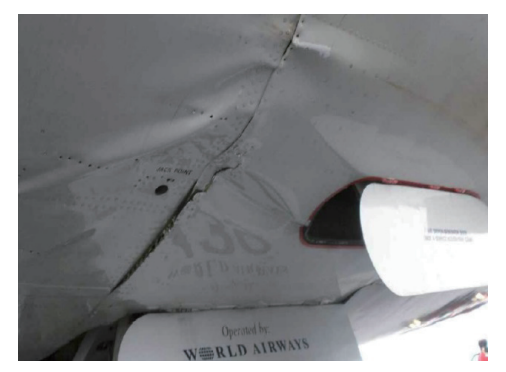

Fig. 2. DC-10 hard-landing accident at Baltimore/Washington International Thurgood Marshall Airport on May 6, 2009. [4] to prevent a serious accident in next flight. And another biggest cause is that there are no objective guidelines as mentioned above. The Boeing Company expects that misjudgments could be decreased to about $30 \%$ if there are objective guidelines based on flight data and about $5 \%$ if structural health monitoring (SHM) and automatic hardlanding determination system would be used [6]. Due to these reasons, a rational and reliable hard-landing detection procedure as SHM technique is essential to guarantee the structural integrity of aircraft.

In order to develop a rational and reliable hard-landing monitoring and detection procedure, landing stage of aircraft operation cycle need to be analyzed accurately by numerical methods so that the decision of hard-landing occurrence can be made and vulnerable area of aircraft to a given hard-landing condition can be identified by using numerical analysis data. For this purpose, an analysis model for efficient and precise landing simulation is required. A full finite element model for nonlinear structural dynamic analysis may be used for aircraft landing analysis, but this approach requires too much computation time to be applied to landing simulation for a practical hard-landing detection procedure. Therefore, an efficient aircraft landing simulation strategy for a hard-landing detection procedure is constructed by using a hierarchical aircraft landing model which consists of a multi-body dynamics (MBD) model equipped with landing gear and tire models, and a finite element model for linear dynamic structural analysis with an extended inertia relief (EIR) method.

In this work, a MBD model for landing simulation which is used to extract the impact forces and inertia forces as well as rigid body motion of aircraft at touch-down is described in section 2, and a finite element model of an aircraft for linear dynamic structural analysis and the EIR method are described in section 3. Landing simulation results by the

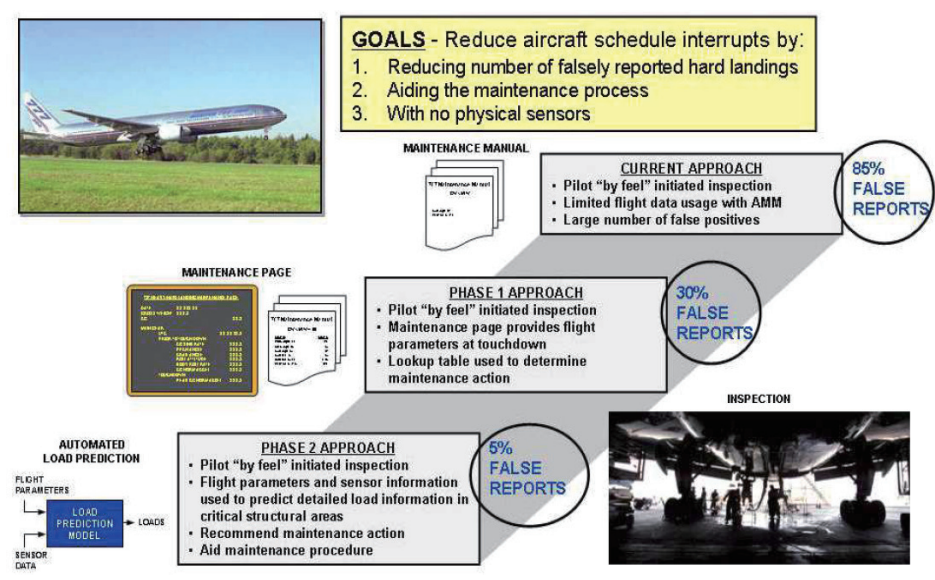

Fig. 3. Effect of development of hard-landing detection technology [6] 
proposed landing simulation technique are presented in section 4 and section 5 concludes this work.

\section{MBD Model for Landing Simulation}

As mentioned above, efficient and precise landing simulation is essential component of aircraft hard-landing monitoring procedure. A hierarchical aircraft landing model is proposed to realize this without full nonlinear structural dynamic analysis. The proposed landing model can reduce computing time and resource considerably. Furthermore, by utilizing MBD to extract the impact forces and inertia forces, it can also simplify the modeling of landing gears which contain oleo dampers and tires which are composed of pneumaticity and rubber.

The hierarchical aircraft landing model proposed in this paper consists of a MBD model and a finite element model for linear dynamic structural analysis. The MBD model contains landing gear and tire models to consider the mechanism of actual landing gears and tires as well as a rigid body aircraft model. By using this MBD model, the impact forces acting on aircraft structures from landing gears and the inertia forces caused by motion of an aircraft can be computed quite accurately. Then, the forces computed by the MBD model as well as the rigid body motion of the aircraft with respect to the center of gravity can be used for linear dynamic structural analysis of a finite element model with the EIR method which will be described in detail in section 3. In this paper, RecurDyn(http://eng.functionbay.co.kr/) is used for MBD analysis and ABAQUS(http://www.3ds.com/ products-services/simulia/products/abaqus/) is used for finite element analysis. The analysis process is illustrated in Fig. 4.

\subsection{Landing Gear Model}

The MBD analysis model of a landing gear is composed of an oleo damper and tires. The internal forces of the oleo damper is composed of hydraulic and pneumatic forces. And tire forces are calculated by using the modified Fiala tire model which is constructed by replacing linear normal forces with non-linear ones. Additionally, spring-backphenomenon is also considered for realistic simulation of landing gears.

\subsubsection{Oleo Damper}

An Oleo damper absorbs shock inside of a landing gear and it can be categorized into single-acting shock absorbers and double-acting shock absorbers. In this paper, a singleacting shock absorber is considered. A single-acting shock absorber is divided into the black upper strut and the slashed lower strut as shown in Fig. 5[7], and the struts are filled with hydraulic fluid and gas. From reference [8], internal force of a single-acting shock absorber can be calculated as summation of hydraulic, pneumatic and friction force.

$$
\begin{aligned}
F_{S} & =p_{h}\left(A_{1}-A_{p}\right)+p_{a}\left(A_{2}-A_{1}\right)+p_{a} A_{p}+F_{f} \\
& =\left(p_{h}-p_{a}\right)\left(A_{1}-A_{p}\right)+p_{a} A_{2}+F_{f} \\
& =\left(p_{h}-p_{a}\right) A_{h}+p_{a} A_{a}+F_{f}
\end{aligned}
$$

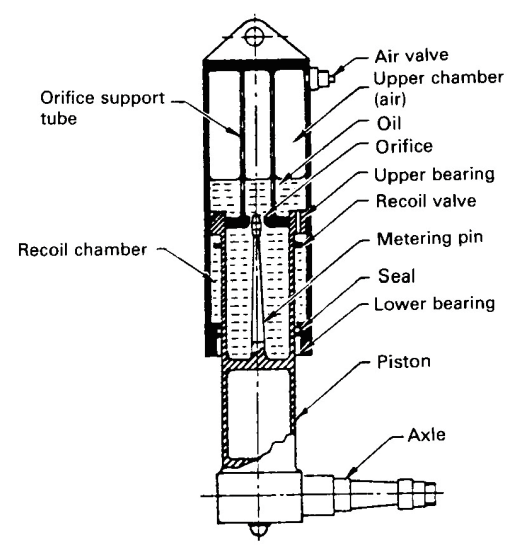

Fig. 5. Single-acting shock absorber [7]

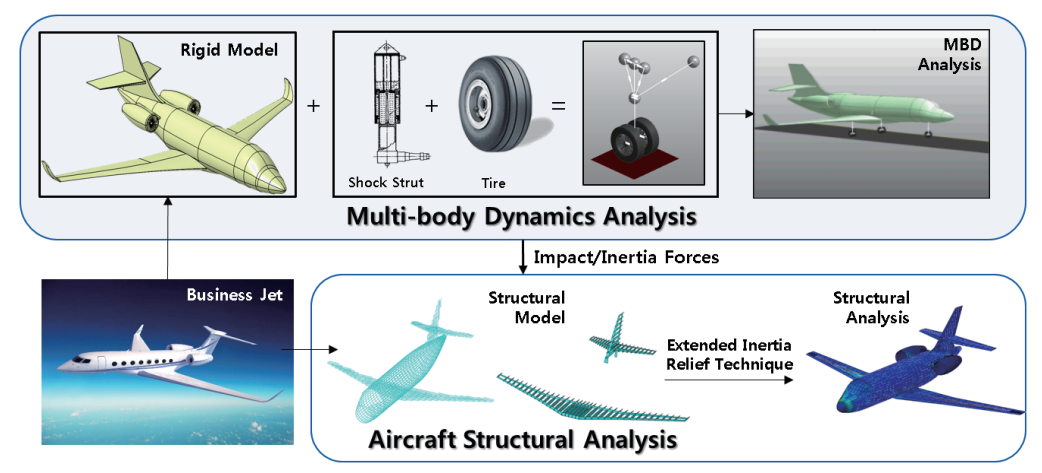

Fig. 4. Conceptual diagram of the hierarchical aircraft landing model 
A conceptual arrangement diagram and a free body diagram (FBD) of a single-acting shock absorber are shown in Fig. 6, 7. From the FBD, the forces generated in struts can be derived as Eqn. (1), (2), and (3) [8]. In Eqn. (3), each term of right-hand side is divided into hydraulic force, pneumatic force and friction force. Among them, friction force is negligible in this mathematical model.

\section{Hydraulic Force}

From reference [8], hydraulic force $\left(F_{h}\right)$ can be expressed as Eqn. (4) by using the Bernoulli's equation and the continuity equation.

$$
F_{h}=\frac{\rho A_{h}^{3}}{2\left(C_{d} A_{n}\right)^{2}} \dot{s}^{2}
$$

From Eqn. (4) hydraulic force is determined by stroke rate $\dot{s}$ and $\dot{s} /|\dot{s}|$ is introduced to identify compression or expansion. Then, the equation of hydraulic force which acts as damping force for stroke can be obtained as Eqn. (5) and its response to stroke rate is shown in Fig. 8.

$$
F_{h}=\frac{\dot{s}}{|\dot{s}|} \frac{\rho A_{h}^{3}}{2\left(C_{d} A_{n}\right)^{2}} \dot{s}^{2}
$$

\section{Pneumatic Force}

Pneumatic pressure of air chamber is determined by polytropic law that is $p_{a} V_{a}^{n}=$ constant. From polytropic law, the final equation of pneumatic force is derived as Eqn. (6)

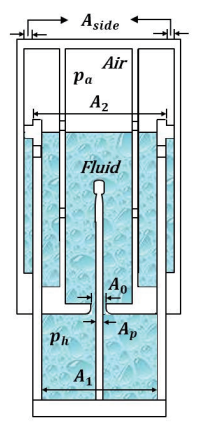

\begin{tabular}{|ll|}
\hline \multicolumn{2}{|c|}{ Symbols } \\
\hline$p_{h}$ & Hydraulic pressure in lower part \\
$p_{a}$ & Pneumatic pressure in upper part \\
$\left(p_{h}-p_{a}\right)$ & Pressure drop across orifice \\
$A_{h}=\left(A_{1}-A_{p}\right)$ & Hydraulic area \\
$A_{a}=A_{2}$ & Pneumatic area \\
$A_{p}$ & Area of metering pin \\
$A_{n} \equiv\left(A_{0}-A_{p}\right)$ & Orifice area \\
$A_{\text {side }}$ & Area of side part \\
$s$ & Stroke of landing gear \\
$V(0)$ & Initial volume of pneumatic part \\
$p_{a}(0)$ & Initial pneumatic pressure \\
$C_{d}$ & Discharge coefficient \\
\hline
\end{tabular}

Fig. 6. Shock absorber arrangement diagram
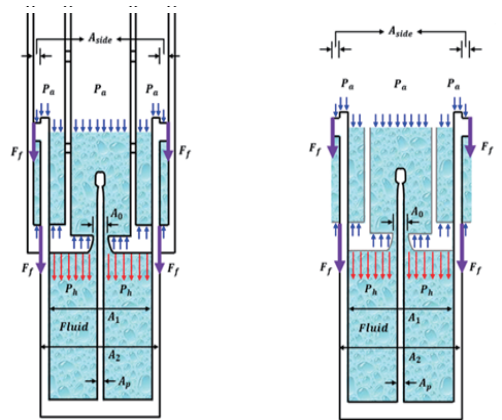

Fig. 7. Shock absorber free body diagram and pneumatic force acts like spring force with respect to stroke as shown in Fig. 9.

$$
F_{a}=p_{a} A_{a}=p_{a}(0) A_{a}\left(\frac{V(0)}{V(0)-A_{a} s}\right)^{n}
$$

\section{Stopper}

When a shock absorber expands completely, the stroke becomes zero and expansion force exists due to air pressure. However, there is no actual expansion because of geometrical constraint. Therefore, in order to deal with this condition, a stopper should be considered which can respond to negative stroke linearly as shown in Fig. 10.

Consequently, the single-acting shock absorber model used in this paper is composed of a spring, a damper and a stopper as shown in Fig. 11.

\subsubsection{Modified Fiala Tire Model}

In this paper, instead of analyzing a full finite element model of a tire, a simplified tire model for MBD simulation

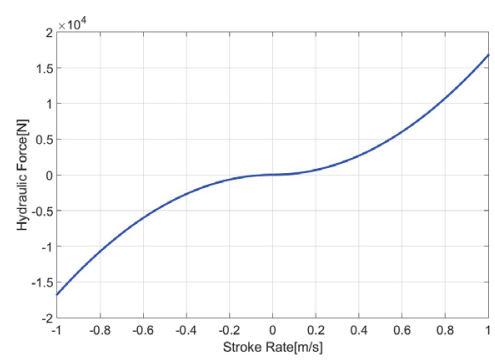

Fig. 8. Hydraulic force

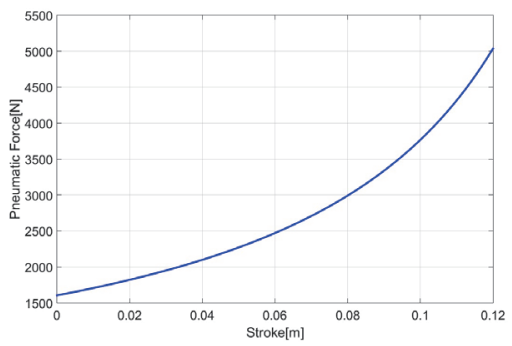

Fig. 9. Pneumatic force

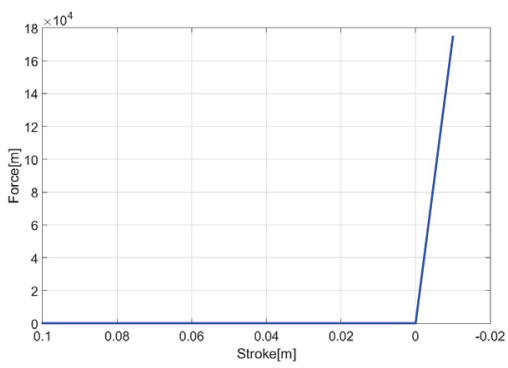

Fig. 10. Stopper 
is used for efficient landing gear simulation. There are various types of simplified tire models for MBD simulation. Among them, the Fiala tire model is selected as an aircraft tire model because it is quite simple and effective in spite of its limitations in describing some physical behaviors such as cornering and handling exactly which are not significant in aircraft landing simulation.

When a tire has vertical deflection, relationship between load and deflection (F-U) is nonlinear due to characteristics of a tire causing nonlinearity such as internal air pressure, nonlinear property of rubber. However, F-U curve of the Fiala tire model in RecurDyn is linear. F-U relation of a tire has considerable influence on the result of aircraft landing simulation. Therefore, as shown in Fig. 12, linear normal force equation of the Fiala tire model in RecurDyn is replaced

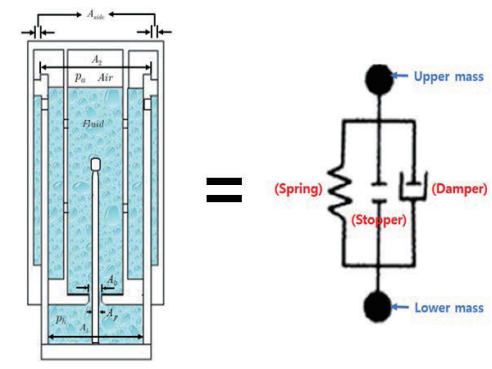

Fig. 11. Conceptual diagram of single-acting shock absorber with nonlinear normal force equation from reference [8] to consider nonlinearity by implementing a user subroutine for RecurDyn, and other equations remain unchanged.

\section{Verification of Landing Gear Model}

To verify the developed user subroutine and landing gear model combined with the shock absorber and the tire model, drop simulation in NACA Report 1154[8] is performed by using RecurDyn and the analysis results are compared with the results in NACA Report 1154. In Fig. 13 and Fig. 14, red solid lines represent results computed by RecurDyn with the developed user subroutine and blue dashed lines represent results from NACA Report. Since the analysis results are in good agreement with those of NACA Report 1154 as shown in Fig. 13 and Fig. 14, validity of landing gear model is confirmed.

\subsubsection{Spring-back Phenomenon}

When an aircraft is landing, spin-up force occurs because of friction between tire and ground. And then spring-back phenomenon occurs due to elasticity of struts as shown in Fig.15[10]. For more realistic analysis, spring-back phenomenon is considered by using a rotational spring.

After rotational stiffness of the rotational spring is calculated by the formula shown in Fig. 16, it is applied to the landing gear model and Fig. 17 shows longitudinal

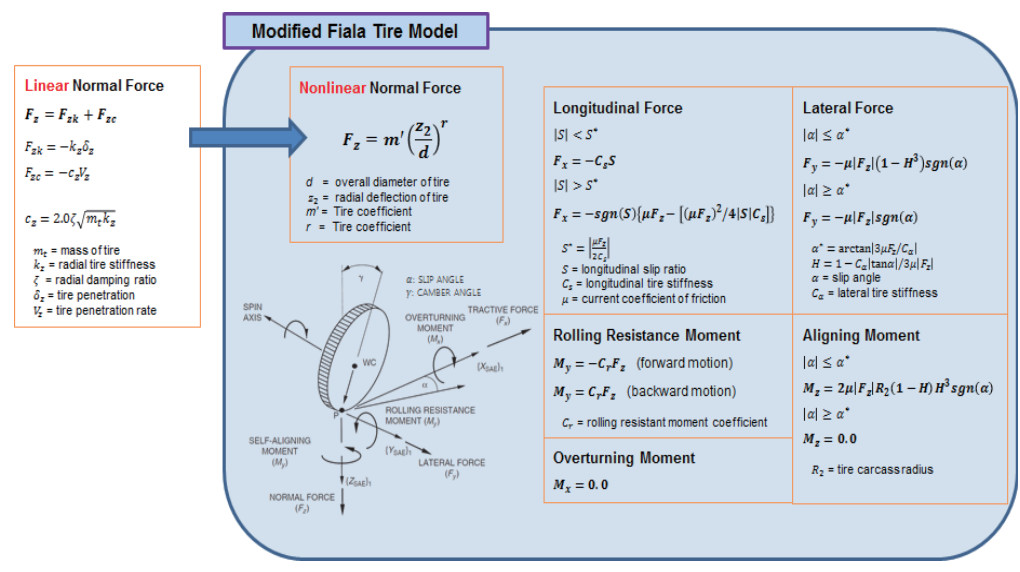

Fig. 12. Formula of modified-Fiala tire model
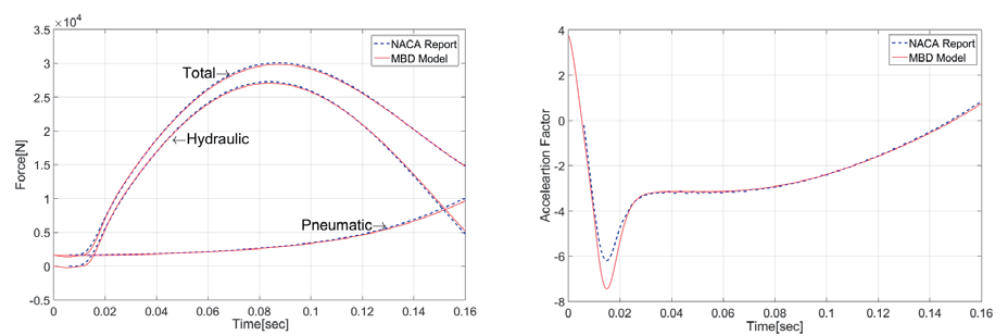

Fig. 13. Comparison of proposed model and NACA Rep.1154 (1/2) 
and vertical forces generated from the main and nose tire respectively. From Fig. 17, one can see that spring-back phenomenon mainly affects longitudinal forces while it has negligible effects on vertical forces.

\subsection{Aircraft Rigid Body Model}

In the hierarchical aircraft landing model proposed in this paper, a MBD model which consists of a rigid airframe model and the landing gear model described above is used to compute the impact forces acting on aircraft structures from landing gears and the inertia forces caused by motion of an aircraft. In order to investigate the validity of this model with rigid airframe, structural analysis is performed for a flexible airframe model which is made up of beam elements as shown in Fig. 18 and the resulting strut forces from landing gears are compared with those computed from the rigid airframe model shown in Fig. 19. As shown in Fig. 20 and Fig. 21, there are no significant differences in strut forces between the flexible and rigid airframe model except for small variations due to vibration of the flexible model, which means the validity of the proposed approach.

The rigid body airframe model has mass and moment of inertia at the position of the center of gravity of an aircraft, and the landing gear models are attached. A business jet aircraft model shown in Fig. 19 is used as a target aircraft for landing simulation and the MBD model for this aircraft is constructed. The solid lines in Fig. 19 represent the position where landing gears are attached. Location of landing gears and extended length of landing gears are based on geometrical information of the target aircraft.

Fig. 22[16] shows the actual shapes of landing gears of
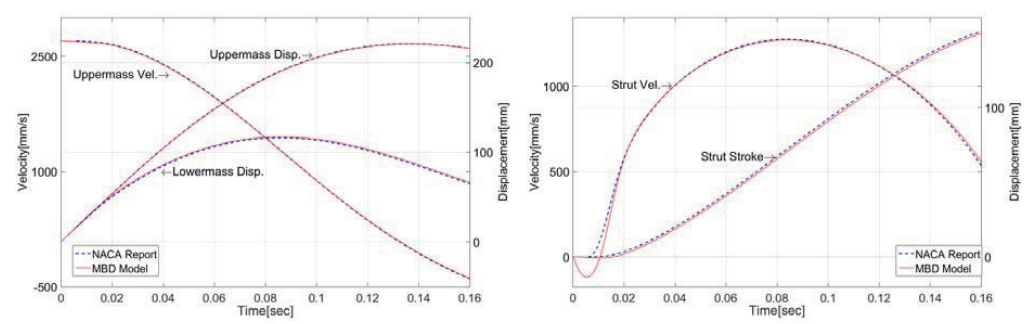

Fig. 14. Comparison of proposed model and NACA Rep.1154 (2/2)
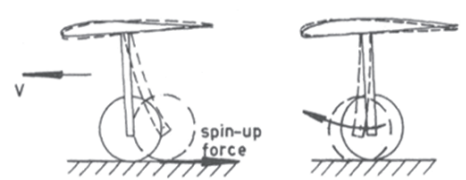

spring-back

Fig. 15. Spin-up and spring back
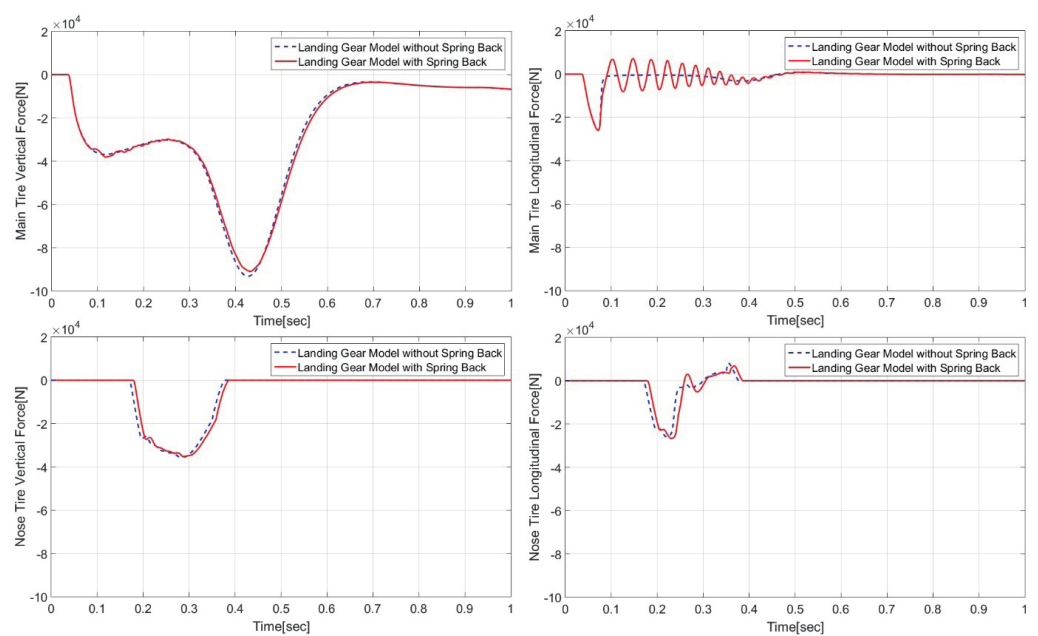

Fig. 17. Effect of spring-back phenomenon to longitudinal and vertical forces 
the target business jet aircraft. In the left of Fig. 22, three pin joints in the main landing gear are illustrated.

In the right of Fig. 22, four pin joints in the nose landing gear are illustrated. Through these pins, landing impact forces are delivered to airframe as 3 force components and 3 moment components at each point.

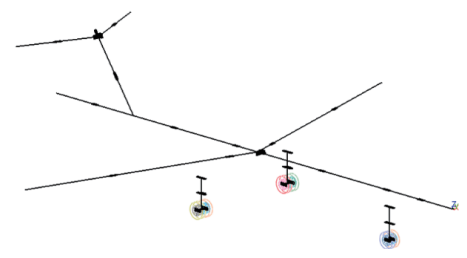

Fig. 18. Flexible airframe model

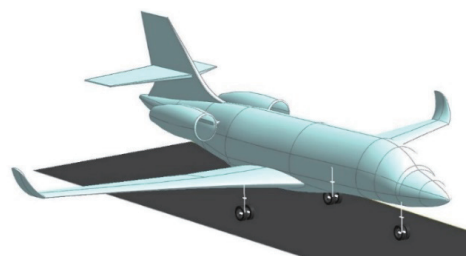

Fig. 19. Rigid airframe model

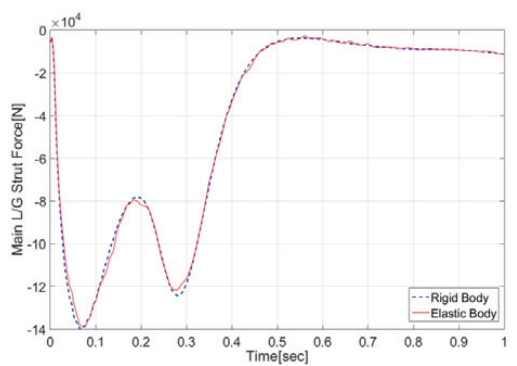

\section{Finite Element Modeling for Structural Analysis}

\subsection{Airframe Structural Model}

In order to analyze structural response of the airframe subject to the impact forces from landing gears and the inertia forces from aircraft motion, a finite element model of the target business jet is constructed as shown in Fig. 23.

Some parts of the aircraft which do not support landing impact forces such as control surfaces and actuators are not considered in the finite element model for simplified modeling and efficient computation. Thus, the finite element model is composed of only structural parts which carry loads. There are spars and ribs which are modelled by shell elements and stringers and stiffeners which are modelled by beam elements in the main and tail wing part. Frames and stringers are modelled by beam elements and the bulkhead consists of shell elements. Skin parts are all made up of shell elements. Engines are not considered as structural parts, so engines are considered as rigid bodies in the structural analysis. The number of elements

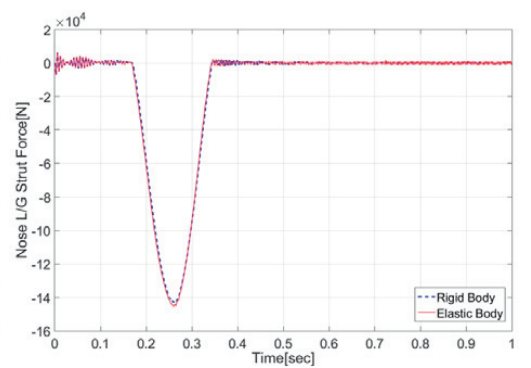

Fig. 20. Strut forces of rigid and flexible airframe model (symmetric approach)
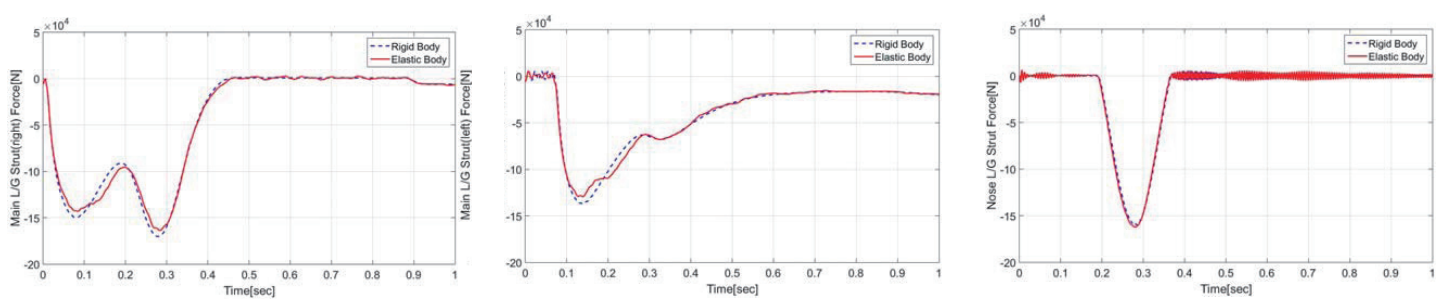

Fig. 21. Strut forces of rigid and flexible airframe model (asymmetric approach)

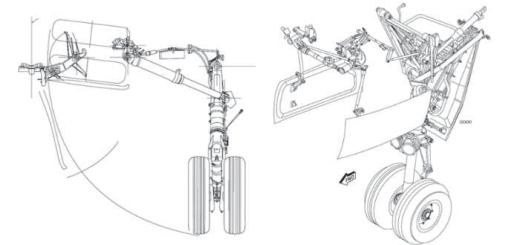

Fig. 22. Simplified feature of main (left) and nose (right) landing gear of target aircraft model [16] and nodes are 4784 and 9279 respectively. In addition to airframe structures, other parts such as actuators, fuel, electronic facilities and payloads are considered as nonstructural mass components and Fig. 24 shows distribution of fuel as an example of non-structural mass. Landing gears are not implemented in the finite element model because they are replaced by the impact loads obtained from the MBD model. 


\subsection{Extended Inertia Relief Technique}

The inertia relief method is extended to arbitrarily moving structures so that it can be applied to aircraft landing simulation of the hierarchical aircraft landing model proposed in this paper. The conventional inertia relief method is usually used to solve linear structural analysis problems for moving bodies in static equilibrium state with constant acceleration as linear static problems by canceling out singularity of stiffness matrix. It can be derived from the equation of motion, Eqn. (7).

$$
\mathbf{M u ̈}+\mathbf{K u}=\mathbf{P}
$$

If displacement $\mathbf{u}$ can be decoupled to rigid body motion and elastic body motion, it can be expressed as the sum of rigid body modes and elastic body modes like Eqn. (8).

$$
\begin{gathered}
\mathbf{u}=\mathbf{u}^{(R)}+\mathbf{u}^{(E)}=\boldsymbol{\Phi}^{(R)} \boldsymbol{\eta}^{(R)}+\boldsymbol{\Phi}^{(E)} \boldsymbol{\eta}^{(E)} \\
\ddot{\mathbf{u}}=\boldsymbol{\Phi}^{(R)} \ddot{\boldsymbol{\eta}}^{(R)}+\boldsymbol{\Phi}^{(E)} \ddot{\boldsymbol{\eta}}^{(E)}=\boldsymbol{\Phi}^{(R)} \ddot{\boldsymbol{\eta}}^{(R)}
\end{gathered}
$$

where $\boldsymbol{\Phi}^{(R)}$ represents rigid body modes and $\boldsymbol{\Phi}^{(E)}$ represents elastic body modes.

Then Eqn. (7) can be rewritten in the following form:

$$
\mathbf{K} \mathbf{u}^{(E)}=\mathbf{P}-\mathbf{M} \boldsymbol{\Phi}^{(R)} \mathbf{m}^{-1}\left[\boldsymbol{\Phi}^{(R)}\right]^{T} \mathbf{P}
$$

where $\mathbf{m}=\left[\boldsymbol{\Phi}^{(R)}\right]^{T} \mathbf{M} \boldsymbol{\Phi}^{(R)}$ and $\mathbf{u}^{(E)}$ is elastic displacement.

Eqn. (10) is the final equation of the conventional inertia relief method. Because singularity of stiffness matrix still exists, boundary condition should be given. If 6 degrees of freedom (DOF) of a node are restrained by boundary condition, the restrained node becomes a reference point and deformation of a body is represented relatively to the reference point. Then, this boundary condition does not cause extra stress, and stress and strain can be obtained by using relative displacement.

The conventional inertia relief method is generally used to take into account the inertia effect of linear acceleration state with no large rotational motion, since it cannot reflect the effects of Coriolis acceleration and centripetal acceleration and it is based on linear system. Therefore, it cannot be applied directly to structural dynamic analysis of landing simulation using prescribed rigid body motion and forces obtained from the MBD model. In order to overcome the limitation of the conventional inertia relief method, principle of virtual work is employed in convective coordinate system. The resulting equation from this variational formulation makes it possible to efficiently consider the inertia forces and deformation dependent on inertia effects corresponding to the prescribed motion including large rotation. This approach is named extended inertia relief (EIR) method. In this approach, a convective coordinate system is introduced in a deformable body to eliminate rigid body motion in nonlinear dynamic analysis with linear elastic deformation. Generally, motion of a deformable body in convective coordinate can be described by rigid body translation, rigid body rotation, and deformation as shown in Fig. 25.

$\mathbf{X}$ is position vector of a point in reference configuration, and $\mathbf{x}(\mathbf{X}, t)$ is the position of $\mathbf{X}$ in current configuration. $\mathbf{x}_{0}(t)$ is the origin of floating coordinate, and $\omega$ is the angular velocity of convective coordinate attached to the body. Then current position vector and acceleration of current position vector can be written as follows [11-13].

$$
\begin{aligned}
\mathbf{x}(\mathbf{X}, t) & =\mathbf{x}_{0}(t)+\left[X_{k}^{t=0} \mathbf{e}_{k}+u_{k}(\mathbf{X}, t) \mathbf{e}_{k}\right] \\
\ddot{\mathbf{x}}(\mathbf{X}, t)= & \ddot{\mathbf{x}}_{0}(t)+\ddot{u}_{k}(\mathbf{X}, t) \mathbf{e}_{k}+\left[X_{k}^{t=0}+u_{k}(\mathbf{X}, t)\right]\left(\dot{\boldsymbol{\omega}} \times \mathbf{e}_{k}\right) \\
& +\left[X_{k}^{t=0}+u_{k}(\mathbf{X}, t)\right] \boldsymbol{\omega} \times\left(\boldsymbol{\omega} \times \mathbf{e}_{k}\right)+2 \dot{u}_{k}(\mathbf{X}, t)\left(\boldsymbol{\omega} \times \mathbf{e}_{k}\right)
\end{aligned}
$$

The meaning of each term in right-hand side of Eq.

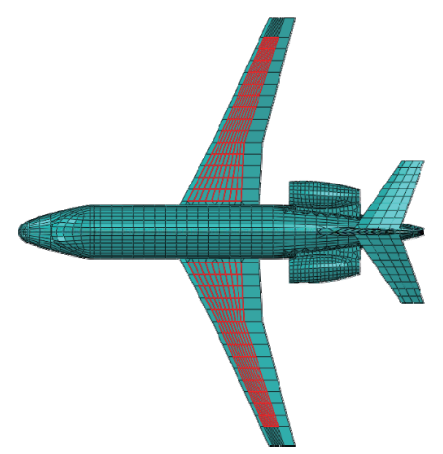

Fig. 24. Distribution of fuel on aircraft wings

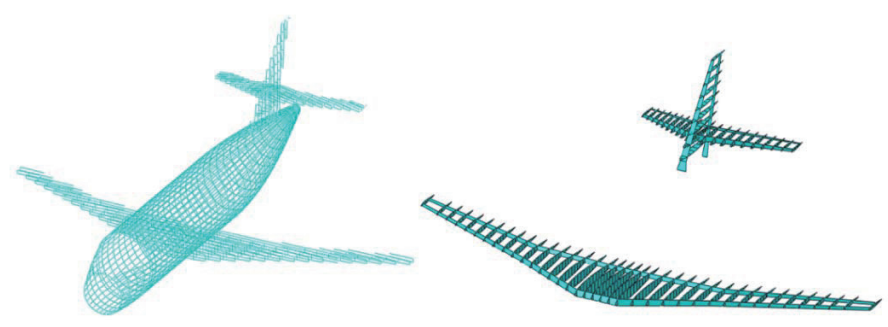

Fig. 23. Airframe finite element model 
(12) is as follows: translational acceleration of rigid body motion, translational acceleration of elastic motion, angular acceleration, centripetal acceleration, and Coriolis acceleration. Here, it is noted that the variation corresponding to the rigid body motion becomes zero since we assume that rigid body motion is already prescribed.

$$
\delta \mathbf{x}=\delta \mathbf{u}+\delta \boldsymbol{\theta} \times\left(X_{k}^{t=0}+u_{k}\right) \mathbf{e}_{k}=\delta \mathbf{x}
$$

By applying principle of virtual work [14],

$$
\delta \mathbf{u} \cdot \int_{V} \rho \ddot{\mathbf{x}} d V+\delta \mathbf{u} \cdot \int_{V} \delta \boldsymbol{\varepsilon}: \boldsymbol{\sigma} d V=\delta \mathbf{u} \cdot \int_{V} \rho \mathbf{f} d V+\delta \mathbf{u} \cdot \int_{\partial V} \mathbf{t} d A
$$

By substituting Eqn. (12) for virtual work term by the inertia forces in Eqn. (13),

$$
\begin{aligned}
\delta \mathbf{u} \cdot \int_{V} \rho \ddot{\mathbf{x}} d V= & \delta \mathbf{u} \cdot \int_{V} \rho \ddot{\mathbf{x}}_{0} d V+\delta \mathbf{u} \cdot \int_{V} \rho \ddot{u}_{k} \mathbf{e}_{k} d V+\delta \mathbf{u} \cdot \int_{V} \rho\left(X_{k}^{t=0}+u_{k}\right)\left(\dot{\boldsymbol{\omega}} \times \mathbf{e}_{k}\right) d V \\
& +\delta \mathbf{u} \cdot \int_{V} \rho\left(X_{k}^{t=0}+u_{k}\right)\left(\boldsymbol{\omega} \times\left(\boldsymbol{\omega} \times \mathbf{e}_{k}\right)\right) d V+2 \delta \mathbf{u} \cdot \int_{V} \rho \dot{u}_{k}\left(\boldsymbol{\omega} \times \mathbf{e}_{k}\right) d V
\end{aligned}
$$

By carrying out finite element discretization for elastic body [15] along with kinematic Eqn. (13) and (14), the following discretized equation can be obtained.

$$
\mathbf{M} \ddot{\mathbf{u}}^{(E)}+2 \mathbf{M} \boldsymbol{\Omega} \dot{\mathbf{u}}^{(E)}+\left(\mathbf{M} \boldsymbol{\Omega} \boldsymbol{\Omega}+\mathbf{M} \dot{\boldsymbol{\Omega}}+\mathbf{K}_{l}\right) \mathbf{u}^{(E)}=\mathbf{P}-\mathbf{M} \ddot{\mathbf{u}}^{(R)}-(\mathbf{M} \boldsymbol{\Omega} \boldsymbol{\Omega}+\mathbf{M} \dot{\boldsymbol{\Omega}}) \mathbf{r}
$$

where $\mathbf{r}=X_{k}^{t=0} \mathbf{e}_{k}$ and $\boldsymbol{\Omega}$ is an angular velocity matrix.

By using this equation, nonlinear structural dynamic problems with large prescribed rigid body motion and small (linear) elastic deformation can be converted to linear structural dynamic problems with inertia forces. Therefore, this approach can considerably increase the efficiency of structural dynamic analysis required for efficient landing simulation.

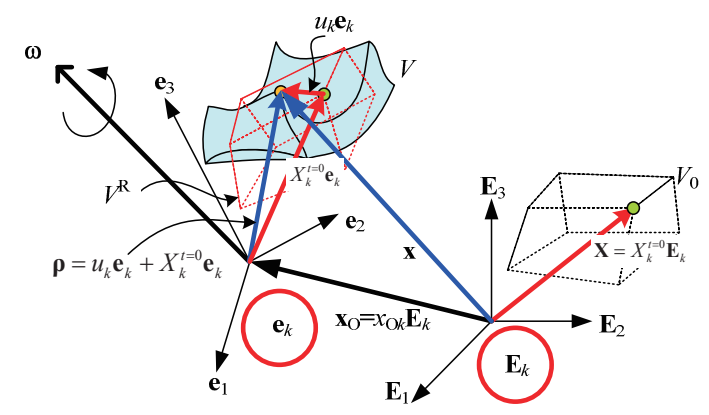

Fig. 25. Motion of elastic body

\subsection{Coupling of MBD Model and Finite Element Model}

The MBD model and finite element model should be combined to perform whole landing simulation required for a hard-landing monitoring procedure. As mentioned above, impact loads and inertia forces as well as rigid body motion are calculated in the MBD model and they are applied to the finite element model as illustrated in Fig. 26. Thus, the coupling between MBD analysis and finite element analysis is one-way coupling because the result of MBD analysis affects finite element analysis while the reverse does not hold. Since only small elastic deformation is considered in the finite element model, one-way coupling is enough for our problem.

Because inertia forces are body forces, they are applied to whole aircraft as translational and rotational acceleration, centripetal acceleration and Coriolis acceleration which are used in the EIR method. And 6 components of the impact loads extracted from landing gears of the MBD model are applied to nodes which are connected to landing gears as concentrated forces and moments. Node positions where the impact loads are applied are marked in Fig. 27.

\section{Simulation of Aircraft Landing}

\subsection{Simulation of MBD Model}

Landing simulation using the hierarchical aircraft landing model proposed in this work is performed to show the efficiency and reliability of the proposed approach. The

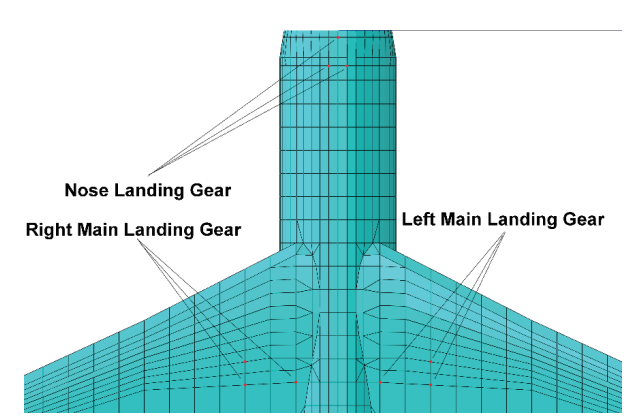

Fig. 27. Node positions where landing gears are connected

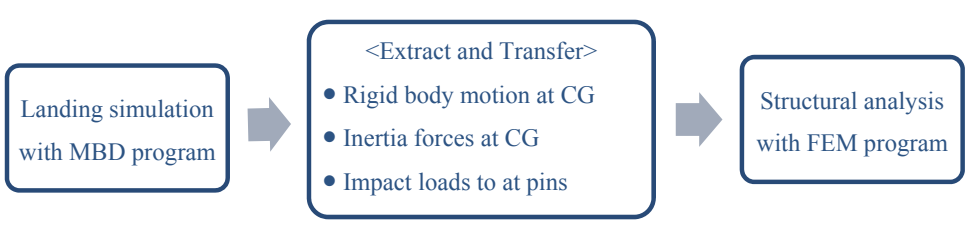

Fig. 26. Block diagram of analysis procedure 
hierarchical aircraft landing model consists of two stages. The first stage is MBD analysis with given touch-down condition, and the second stage is finite element analysis with the EIR method using rigid body motion and forces computed from the first stage.

In order to perform MBD analysis for landing simulation, initial landing condition which is a major factor to determine the occurrence of hard-landing is constructed as shown in Tab. 1. This is asymmetrical landing condition which causes asymmetrical landing of the aircraft.

Using initial condition listed in Tab. 1, landing simulation is carried out with the MBD model of the business jet aircraft. The MBD model of the aircraft is composed of rigid airframe expressed as point mass and landing gear models which implement tires and actuators. Fig. 28 shows the sequence of asymmetrical landing of the aircraft obtained from the result of MBD analysis with the initial landing condition in Tab. 1.

\subsection{Simulation of Finite Element Model}

The loads obtained from MBD analysis are applied to the finite element model and structural analysis is performed. Structural analysis for aircraft landing simulation need to be carried out for a nonlinear finite element model coupled with detail landing gear models in order to obtain highly accurate results because aircraft landing behavior can be considered as geometrically nonlinear due to large rotation

Table 1. Touch-down conditions

\begin{tabular}{|c|c|c|}
\hline \multirow{3}{*}{ Approaching angle } & Roll & $3^{\circ}$ \\
\hline & Pitch & $3^{\circ}$ \\
\hline & Yaw & $0^{\circ}$ \\
\hline Approaching velocity & \multicolumn{2}{|c|}{$97.20 \mathrm{knots}(=50.00 \mathrm{~m} / \mathrm{s})$} \\
\hline Forward movement velocity & \multicolumn{2}{|c|}{$50 \mathrm{~m} / \mathrm{s}$} \\
\hline Downward movement velocity & \multicolumn{2}{|c|}{$0.5 \mathrm{~m} / \mathrm{s}$} \\
\hline
\end{tabular}

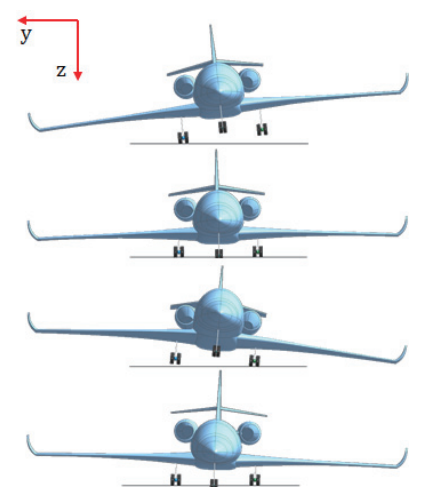

Fig. 28. Sequential result of landing simulation of airframe and coupling between rigid body motion and structural deformation. However, it is not feasible for landing simulation as a part of a hard-landing monitoring procedure because nonlinear analysis is highly time consuming task. Therefore, a methodology is proposed which can replace nonlinear analysis by linear analysis with comparable accuracy and much higher efficiency. Linear finite element analysis with the EIR method using the results from MBD analysis is the methodology proposed in this paper, and the efficiency and reliability of the proposed methodology is confirmed by comparing the result of nonlinear analysis with that of linear analysis with the EIR method.

\subsubsection{Nonlinear Analysis of Finite Element Model}

In order to obtain reference data for comparison with the result of the proposed approach, nonlinear structural dynamic analysis is carried out for aircraft landing simulation. Only geometrical nonlinearity for the finite element model is considered. Instead of including detail nonlinear finite element models of landing gears coupled with airframe, they are replaced by impact loads obtained from MBD analysis. The MBD model described in section 2 is used to compute the impact forces acting on connection points of landing gears to airframe and the computed impact forces are applied to the nonlinear finite element model of airframe as external forces. Since large rotation of the aircraft is considered by the geometrical nonlinearity of the finite element model, prescribed motion obtained from MBD analysis to compute inertia effects are not required for nonlinear finite element analysis while linear finite element analysis with the EIR method requires this information.

As initial condition for reference nonlinear analysis, asymmetrical touch-down condition presented in Tab. 1 is used. The analysis result at 0.25 seconds is shown in Fig. 29.

In Fig. 29, the element which has maximum stress and the magnitude of the maximum stress in the left side box need to be noted for comparison. The analysis running time is about 31 hours.

\subsubsection{Linear Analysis of Finite Element Model}

Linear structural dynamic analysis with the EIR method proposed in this research is performed. The EIR method requires prescribed motion such as translational acceleration, angular velocity and angular acceleration at each node as well as the impact loads from landing gears.

In landing simulation by the proposed EIR method, the landing impact loads obtained from MBD analysis are applied to the linear finite element model of airframe as external forces. These external forces are included in force vector $\mathbf{P}$ in Eqn. (15). And the prescribed motion of the 
aircraft obtained by MBD analysis is utilized to compute inertia effects in Eqn. (15).

The analysis result at 0.25 seconds by linear analysis is shown in Fig. 30.

In Fig. 30, the element which has maximum stress and the magnitude of the maximum stress in the left side box need to be noted for comparison with the previous nonlinear analysis result. The analysis running time is about 25 minutes.

\subsection{Comparison of Analysis Results}

The structural behavior obtained by the EIR method is compared with that from the nonlinear transient dynamic analysis. Tab. 2 enumerates data about maximum stress points with respect to time. These data include initial positions of nodes with maximum stress, node indices of those nodes, and magnitudes of maximum stress obtained
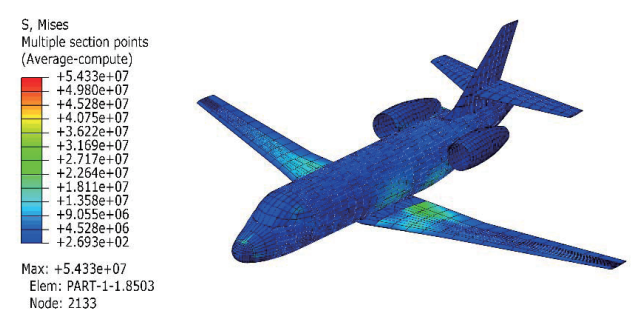

Fig. 29. Result of general nonlinear dynamic analysis at $0.25 \mathrm{~s}$
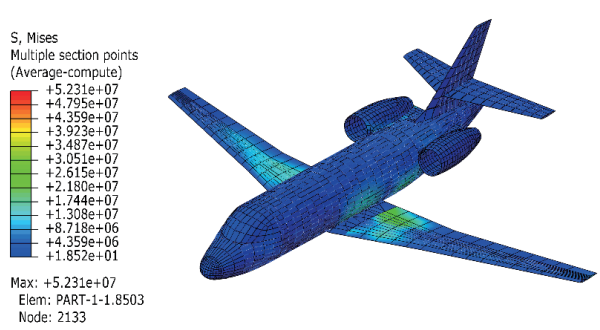

Fig. 30. Result of linear dynamic analysis with EIR method at $0.25 \mathrm{~s}$ from supporting structures of each finite element model. As shown in Tab.2, the results by linear analysis with the EIR method are in good accordance with those by nonlinear analysis.

Although small differences are found in magnitude of maximum stresses in Tab. 2, these differences can be considered to be negligible for the aim of this simulation. Therefore, it can be concluded that linear analysis with the EIR method has enough accuracy as a component of a hardlanding detection procedure. When it comes to efficiency or performance, linear analysis with the EIR method is much superior to nonlinear analysis as shown in Tab. 3. The analysis time for linear analysis with the EIR method is about 75 times faster than that of nonlinear analysis.

Figure 31, 32 and 33 show that stress spectrums at the structures of the aircraft are nearly the same when the maximum landing force is applied to each landing gear. In these figures, the left figures represent the results from nonlinear analysis while the right ones represent the results from linear analysis with the EIR method.

From these comparison results, one can confirm the efficiency and accuracy of the proposed method.

\section{Conclusion}

Although hard-landing is the most common accident among various types of accidents, there are no objective

Table 3. Analysis running time

\begin{tabular}{cc}
\hline Analysis type & Analysis time \\
\hline Nonlinear analysis & $114,091 \mathrm{sec}$ \\
\hline Linear analysis with EIR method & $1,523 \mathrm{sec}$ \\
\hline
\end{tabular}

Table 2. Comparison of maximum stress results from nonlinear analysis and linear analysis

\begin{tabular}{|c|c|c|c|c|}
\hline \multicolumn{2}{|c|}{$\begin{array}{c}\text { Maximum Stress } \\
\text { Position / Magnitude }\end{array}$} & Nonlinear analysis & Linear analysis with EIR & $\begin{array}{l}\text { Distance }(\mathrm{m}) / \\
\text { Relative error }\end{array}$ \\
\hline \multirow{2}{*}{$t=0.25 \mathrm{~s}$} & Position & $\begin{array}{c}-11.0387,-1.6144,1.6538 \\
\text { (No. 2133) }\end{array}$ & $\begin{array}{c}-11.0387,-1.6144,1.6538 \\
\text { (No. 2133) }\end{array}$ & 0.00 \\
\hline & Magnitude & $35.88 \mathrm{MPa}$ & $34.54 \mathrm{MPa}$ & $3.73 \%$ \\
\hline \multirow{2}{*}{$t=0.50 \mathrm{~s}$} & Position & $\begin{array}{c}-16.9971,-0.6404,0.0508 \\
\text { (No. 2428) }\end{array}$ & $\begin{array}{c}-16.9971,-0.6404,0.0508 \\
\text { (No. 2428) }\end{array}$ & 0.00 \\
\hline & Magnitude & $32.87 \mathrm{MPa}$ & $31.98 \mathrm{MPa}$ & $2.70 \%$ \\
\hline \multirow{2}{*}{$t=0.75 \mathrm{~s}$} & Position & $\begin{array}{c}-16.9971,-0.6404,0.0508 \\
\text { (No. 2428) }\end{array}$ & $\begin{array}{c}-16.9971,-0.6404,0.0508 \\
\text { (No. 2428) }\end{array}$ & 0.00 \\
\hline & Magnitude & $38.30 \mathrm{MPa}$ & $39.12 \mathrm{MPa}$ & $2.14 \%$ \\
\hline \multirow{2}{*}{$t=0.99 \mathrm{~s}$} & Position & $\begin{array}{c}-16.1127,-0.0646,-0.6256 \\
\text { (No.1520) }\end{array}$ & $\begin{array}{c}-16.1119,0.2178,-0.6108 \\
(\text { No.1521) }\end{array}$ & 0.28 \\
\hline & Magnitude & $17.94 \mathrm{MPa}$ & $17.38 \mathrm{MPa}$ & $3.12 \%$ \\
\hline
\end{tabular}


guidelines to determine the occurrence of hard-landing. With a view to establishing a rational hard-landing decision procedure, a new analysis model for efficient landing simulation is proposed by replacing general nonlinear landing simulation with MBD and linear structural analysis.

In this work, an analysis model for MBD analysis which consists of landing gear and tire models is constructed to extract inertia forces and impact forces as well as rigid body motion of aircraft. The equation for landing gear in this MBD model is derived as a function of normal direction deflection and velocity, and it is verified. The equation for tires in this MBD model is also derived for the modified Fiala tire model that shows nonlinear behavior. The EIR method is proposed for efficient structural analysis. By using the EIR method presented in this work, nonlinear structural dynamic analysis for landing simulation can be replaced successfully by linear structural dynamic analysis with prescribed motion and forces obtained by MBD analysis.

By using example touch-down condition as initial condition for landing simulation, both nonlinear dynamic analysis and the proposed linear dynamic analysis with the EIR method are performed, and the results show that the proposed analysis method can produce numerical analysis result with acceptable accuracy and has much better performance than nonlinear dynamic analysis. Therefore, it can be concluded that the proposed aircraft landing model is very efficient and reliable as a component of a hard-landing monitoring procedure.

\section{Acknowledgment}

This work was partially supported by INHA UNIVERSITY Research Grant.

\section{References}

[1] Boeing, "Statistical Summary of Commercial Jet Airplane Accidents", Boeing Commercial Airplanes, 2003.

[2] Yong-Kyu Oh, Sang-Ki Sim, and Ki-Su Shin, "A Study of the Effects of Hard Landing on Aircraft Structure", Journal of A Korea Institute of Military Science and Technology, Vol. 14, No. 5, 2011, pp. 805- 811.

[3] http://www.flightstory.net/20120620/ana-b767-damaged -in-hard-landing-videos/

[4] https://aviationsafetynetwork.wordpress.com/2010/ 03/11/ntsb-releases-docket-on-dc-10-hard-landing-accident/

[5] Flight Safety Foundation Editorial Staff, "Stabilized Approach and Flare are Keys to Avoiding Hard Landings",
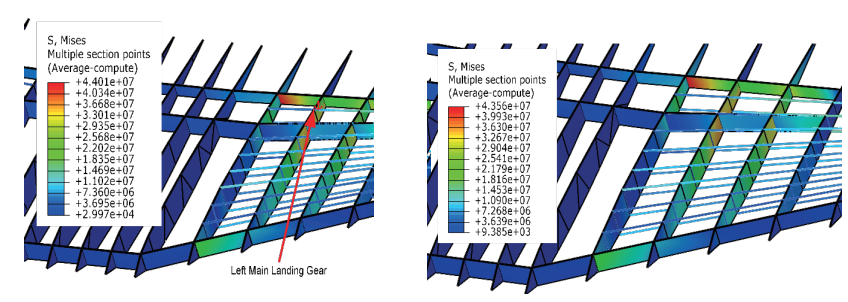

Fig. 31. Comparison of stress spectrum at maximum load in left landing gear (0.27s)
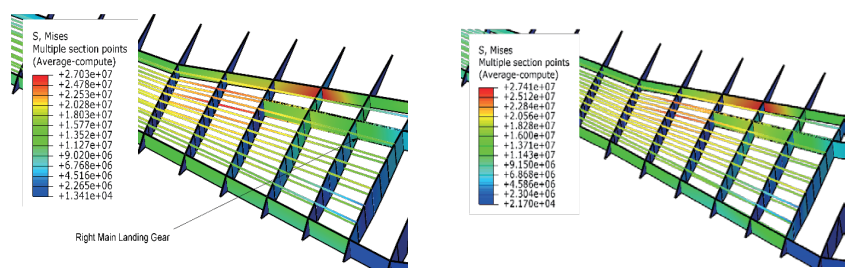

Fig. 32. Comparison of stress spectrum at maximum load in right landing gear (0.61s)
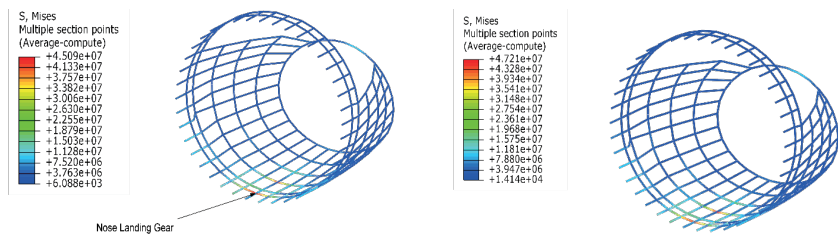

Fig. 33. Comparison of stress spectrum at maximum load in nose landing gear (0.64s) 
Flight Safety Digest, Vol. 23,. No. 8, 2004, pp.1-25.

[6] Young, J., Haugse, E. and Davis, C., "Structural Health Management an Evolution in Design", Structural Health Monitoring 2009: From System Integration to Autonomous Systems, Proceedings of 7th International Workshop on Structural Health Monitoring, Stanford, CA, 2009, pp.3-13.

[7] Norman S., Currey, Aircraft Landing Gear Design: Principles and Practices, AIAA Education Series, Ohio, 1988.

[8] Benjamin Milwitzky, and Francis E. Cook, "Report 1154-Analysis of Landing-Gear Behavior", National Advisory Committee for Aeronautics.

[9] Mike Blundell, and Damian Harty, The Multibody Systems Approach to Vehicle Dynamics, Michael Blundell and Damian Harty, Burlington, 2004.

[10] Jacob Ijff, "Analysis of dynamic aircraft landing loads, and a proposal for rational design landing load requirements",
A degree of Doctor, Delft University of Technology, 1972.

[11] Greenwood, Classical Dynamics, Dover Publications, New York, 1977.

[12] L.G.Kraige and J.L.Meriam, Kraige, L. G. and Meriam, J. L., Engineering Mechanics Dynamics, 6th edition, WILEY, Hoboken, 2008.

[13] MARION, Classical Dynamics of Particles and Systems, 2nd edition, University of Maryland.

[14] Kyuichiro Washizu, Variational Methods in Elasticity and Plasticity, The second Eds., Pergamon Press LTD, New York, 1975.

[15] K.K.GUPTA, J.L.MEEK, MEEK, J. L. and GUPTA, K.K., Finite Element Multidisciplinary Analysis, AIAA EDUCATION SERIES, Washington, DC, 2003.

[16] http://code7700.com/g450_landing_gear_and_doors. html 